\title{
SPECTRAL DECOMPOSITION OF REAL SYMMETRIC QUADRATIC $\lambda$-MATRICES AND ITS APPLICATIONS
}

\author{
MOODY T. CHU AND SHU-FANG XU
}

\begin{abstract}
Spectral decomposition provides a canonical representation of an operator over a vector space in terms of its eigenvalues and eigenfunctions. The canonical form often facilitates discussions which, otherwise, would be complicated and involved. Spectral decomposition is of fundamental importance in many applications. The well-known GLR theory generalizes the classical result of eigendecomposition to matrix polynomials of higher degrees, but its development is based on complex numbers. This paper modifies the GLR theory for the special application to real symmetric quadratic matrix polynomials, $\mathcal{Q}(\lambda)=M \lambda^{2}+C \lambda+K, M$ nonsingular, subject to the specific restriction that all matrices in the representation be real-valued. It is shown that the existence of the real spectral decomposition can be characterized through the notion of real standard pair which, in turn, can be constructed from the spectral data. Applications to a variety of challenging inverse problems are discussed.
\end{abstract}

\section{INTRODUCTION}

Representing an operator in terms of its eigenvalues and eigenvectors, the socalled spectral decomposition, has been a powerful tool in many disciplines of sciences and engineering. The spectral decomposition of a single matrix, often expressed in the Jordan canonical form, has significant applications such as simplifying the representation of complicated systems, shedding light on the asymptotic behavior of differential equations, or characterizing the performance behavior of numerical algorithms [13, 15. This paper concerns itself with the spectral decomposition of a special class of quadratic $\lambda$-matrices,

$$
\mathcal{Q}(\lambda):=M \lambda^{2}+C \lambda+K
$$

where $M, C$ and $K$ are symmetric matrices in $\mathbb{R}^{n \times n}$ and $M$ is nonsingular.

Real symmetric quadratic $\lambda$-matrices arise frequently in the study of applied mechanics, electrical oscillation, vibro-acoustics, fluid dynamics, signal processing, and finite element model of some critical PDEs [18, 28]. The specifications of the underlying physical system are embedded in the matrix coefficients $(M, C, K)$. Typically, the forward analysis involves finding scalars $\lambda \in \mathbb{C}$ and nonzero vectors $\mathbf{x} \in \mathbb{C}^{n}$, called the eigenvalues and eigenvectors of the system, respectively, to satisfy

Received by the editor March 28, 2007 and, in revised form, December 12, 2007.

2000 Mathematics Subject Classification. Primary 65F15, 15A22, 65F18, 93 B55.

Key words and phrases. Quadratic $\lambda$-matrix, spectral decomposition, real standard pair, inverse eigenvalue problem.

Research of the first author was supported in part by the National Science Foundation under grants DMS-0505880 and CCF-0732299.

Research of the second author was supported in part by NSFC under grant 10571007 .

(c)2008 American Mathematical Society Reverts to public domain 28 years from publication 
the algebraic equation $Q(\lambda) \mathbf{x}=0$. Our goal is to characterize a representation of the coefficient matrices $(M, C, K)$ in terms of the spectral data of $\mathcal{Q}(\lambda)$. The emphasis in this discussion is that all information involved in the representation must be real-valued. The efficacy of our theory will be demonstrated by its application to some challenging inverse problems at the end of this paper.

To motivate our consideration and set the notation for later comparison, recall first the classical spectral decomposition of a symmetric matrix $A \in \mathbb{R}^{n \times n}$,

$$
A=X \Lambda X^{\top}
$$

where $X=\left[\mathbf{x}_{1}, \ldots, \mathbf{x}_{n}\right] \in \mathbb{R}^{n \times n}$ and $\Lambda=\operatorname{diag}\left\{\lambda_{1}, \ldots, \lambda_{n}\right\} \in \mathbb{R}^{n \times n}$ are matrices composed of orthonormal eigenvectors $\mathbf{x}_{i}$ and corresponding eigenvalues $\lambda_{i}$, respectively. It is important to point out that the orthogonality $X^{\top} X=I_{n}$ of the eigenvector matrix $X$ is an intrinsic property due to the symmetry of $A$, which will be explored later. Consider next the linear pencil

$$
\mathcal{L}_{0}(\lambda)=B \lambda-A,
$$

where $A$ and $B$ are symmetric matrices in $\mathbb{R}^{n \times n}$ and $B$ is positive definite. Again, the coefficients $(B, A)$ of the pencil can be represented as

$$
\left\{\begin{array}{l}
B=X^{-\top} X^{-1} \\
A=X^{-\top} \Lambda X^{-1}
\end{array}\right.
$$

where $X=\left[\mathbf{x}_{1}, \ldots, \mathbf{x}_{n}\right]$ and $\Lambda=\operatorname{diag}\left\{\lambda_{1}, \ldots, \lambda_{n}\right\}$, satisfying $A \mathbf{x}_{i}=\lambda_{i} B \mathbf{x}_{i}$, are still real matrices. It is in this sense that we call (1.4) the spectral decomposition of the linear pencil $(B, A)$. Note that the "orthogonality" of $X$ is built in the first equation in (1.4) which, called $B$-orthogonality, can be written as $X^{\top} B X=I_{n}$. Even without the positive definiteness of $B$, a real symmetric pencil $B \lambda-A$ can still be reduced to the diagonal form $I \lambda-\Lambda$ by congruence under mild conditions such as all eigenvalues being simple, except that the nonsingular matrix $X$ usually is complexvalued [10, 15]. (A short proof can also be found in [4 and [19, Appendix A]. A more complete and systematic development is made in [23.) If the transformation is limited to real congruence, then the diagonal form should be replaced by a block diagonal form with at most $2 \times 2$ blocks along the diagonal [12, Theorem 1.5.4]. See also 24 for a different scenario, where $B$ is real symmetric and $A$ is real skewsymmetric. In this paper we ask whether a similar notion can be generalized to the real symmetric quadratic $\lambda$-matrix $\mathcal{Q}(\lambda)$ where three coefficient matrices $(M, C, K)$ are to be decomposed simultaneously.

The query we are asking is a classical problem already considered in the seminal book by Gohberg, Lancaster and Rodman 11. Indeed, via the notion of Jordan triple the GLR theory developed in [11] works for general matrix polynomials of arbitrary degrees. If the coefficients of the matrix polynomial are self-adjoint, then additional properties such as the existence of a self-adjoint triple and the sign characteristic can be developed from the theory [11, Chapter 10]. As an example, we mention a case studied in [22] for a real symmetric quadratic $\lambda$-matrix $\mathcal{Q}(\lambda)$ under the assumption that all eigenvalues are simple and nonreal. Let $X_{c}=\left[\mathbf{x}_{1}, \ldots, \mathbf{x}_{n}\right]$ and $\Lambda=\operatorname{diag}\left\{\lambda_{1}, \ldots, \lambda_{n}\right\}$ denote $n \times n$ matrices of (half of) the eigenvectors and the eigenvalues, respectively, where $\lambda_{i}$ are those eigenvalues residing in the upper 
half-complex plane. Define

$$
\begin{aligned}
\mathscr{X} & :=\left[X_{c}, \overline{X_{c}}\right] \in \mathbb{C}^{n \times 2 n}, \\
\mathscr{T} & :=\left[\begin{array}{cc}
\Lambda & 0 \\
0 & \bar{\Lambda}
\end{array}\right] \in \mathbb{C}^{2 n \times 2 n} .
\end{aligned}
$$

The GLR theory [22, Theorem 2] asserts that if the matrix

$$
\mathscr{U}:=\left[\begin{array}{c}
\mathscr{X} \\
\mathscr{X} \mathscr{T}
\end{array}\right] \in \mathbb{C}^{2 n \times 2 n}
$$

is nonsingular (so that $(\mathscr{X}, \mathscr{T})$ forms a complex Jordan pair), then the eigenvector matrix $\mathscr{X}$ enjoys the "orthogonality",

$$
\mathscr{X} \mathscr{X}^{\top}=0,
$$

and the matrix coefficients $(M, C, K)$ can be decomposed as

$$
\left\{\begin{aligned}
M & =\left(\mathscr{X} \mathscr{T} \mathscr{X}^{\top}\right)^{-1}, \\
C & =-M\left(\mathscr{X}^{2} \mathscr{X}^{\top}\right) M \\
K & =-\left(\mathscr{X} \mathscr{T}^{-1} \mathscr{X}^{\top}\right)^{-1} .
\end{aligned}\right.
$$

The challenge is that, even with symmetry, a real symmetric matrix polynomial often has complex-valued eigenstructure [10]. The Jordan pair $(\mathscr{X}, \mathscr{T})$ constructed above by the GLR theory for the spectral decomposition (1.9) is complex-valued. A real-valued decomposition for the real coefficients of a quadratic $\lambda$-matrix in the same spirit of (1.4) for a linear pencil is not obvious. One of our contributions is to fill in the details of a real-valued spectral decomposition. Additionally, we show that the real-valued spectral decomposition of $\mathcal{Q}(\lambda)$ naturally carries a specially structured scaling factor which we could consider as a "parameter". Its simplest form helps to facilitate the study of some inverse problems. Our main thrust in this paper is to investigate these properties and to demonstrate some interesting applications.

\section{Spectral Decomposition}

Linearization is a rather convenient way to cast a matrix polynomial as a linear problem [14. One special way to linearize the real symmetric quadratic $\lambda$-matrix $\mathcal{Q}(\lambda)$ is the linear pencil,

$$
\mathcal{L}(\lambda):=\mathcal{L}(\lambda ; M, C, K)=\left[\begin{array}{cc}
C & M \\
M & 0
\end{array}\right] \lambda-\left[\begin{array}{cc}
-K & 0 \\
0 & M
\end{array}\right] .
$$

It is easy to see that $\mathcal{Q}(\lambda)$ and $\mathcal{L}(\lambda)$ are equivalent in the sense that $Q(\lambda) \mathbf{x}=0$ if and only if

$$
\left(\left[\begin{array}{cc}
C & M \\
M & 0
\end{array}\right] \lambda-\left[\begin{array}{cc}
-K & 0 \\
0 & M
\end{array}\right]\right)\left[\begin{array}{l}
\mathbf{x} \\
\mathbf{y}
\end{array}\right]=0
$$

Because we have assumed that $M$ is nonsingular, we even know that $\mathbf{y}=\lambda \mathbf{x}$. Clearly, $\mathcal{L}(\lambda)$ is real symmetric. Note, however, that the leading coefficient $\left[\begin{array}{cc}C & M \\ M & 0\end{array}\right]$ of $\mathcal{L}(\lambda)$ is not positive definite, so the decomposition for $\mathcal{Q}(\lambda)$ cannot be answered directly in the same way as the decomposition (1.4) for $\mathcal{L}_{0}(\lambda)$.

At present, we have not yet assumed any eigenstructure of $\mathcal{Q}(\lambda)$. It is entirely possible that the eigenvalues are complex-valued or have high geometric multiplicities. The notion of standard pair introduced in 11] will be a fundamental tool for our development. The canonical form of a standard pair can be considered as 
a generalization of the Jordan canonical form to matrix polynomials. So that this paper is self-contained, we briefly review the ideas below, but limit ourselves to the special class of real symmetric quadratic polynomials (1.1) and require specifically that all matrices involved be real-valued. From time to time, we shall compare our real formulation with its counterpart of complex formulation already developed in [11] and point out the difference when the demand for real-valued matrices is enforced.

The symmetry of the matrix coefficients is not essential in the following definition but we insist that $M$ must be nonsingular.

Definition 2.1. A pair of matrices $(\mathfrak{X}, \mathfrak{T}) \in \mathbb{R}^{n \times 2 n} \times \mathbb{R}^{2 n \times 2 n}$ is called a real standard pair for the quadratic $\lambda$-matrix $\mathcal{Q}(\lambda)$ if and only if the matrix

$$
U=U(\mathfrak{X}, \mathfrak{T}):=\left[\begin{array}{c}
\mathfrak{X} \\
\mathfrak{X T}
\end{array}\right]
$$

is nonsingular and the equation

$$
M \mathfrak{X} \mathfrak{T}^{2}+C \mathfrak{X T}+K \mathfrak{X}=0
$$

holds.

The existence of a real standard pair for $\mathcal{Q}(\lambda)$ is clear. Direct computation shows that the special choice $\left(\mathfrak{X}_{0}, \mathfrak{T}_{0}\right)$ with

$$
\left\{\begin{aligned}
\mathfrak{X}_{0} & :=\left[I_{n}, 0\right], \\
\mathfrak{T}_{0} & :=\left[\begin{array}{cc}
0 & I \\
-M^{-1} K & -M^{-1} C
\end{array}\right],
\end{aligned}\right.
$$

is a real standard pair of $\mathcal{Q}(\lambda)$. The problem is that the self-reference of $\left(\mathfrak{X}_{0}, \mathfrak{T}_{0}\right)$ to the original matrix coefficients makes this pair not very useful in practice. We seek real standard pairs that contain the eigeninformation more explicitly. We shall construct such a real standard pair later, but first we point out the following result that shows how two arbitrary real standard pairs are related to each other. The proof follows directly from [11, Theorem 1.25].

Lemma 2.2. Let $\left(\mathfrak{X}_{1}, \mathfrak{T}_{1}\right)$ be a given real standard pair of $\mathcal{Q}(\lambda)$. Then $\left(\mathfrak{X}_{2}, \mathfrak{T}_{2}\right)$ is also a real standard pair of $\mathcal{Q}(\lambda)$ if and only if

$$
\left\{\begin{array}{l}
\mathfrak{X}_{2}=\mathfrak{X}_{1} \mathfrak{S}, \\
\mathfrak{T}_{2}=\mathfrak{S}^{-1} \mathfrak{T}_{1} \mathfrak{S},
\end{array}\right.
$$

for some $2 n \times 2 n$ real invertible matrix $\mathfrak{S}$. In this case, $\mathfrak{S}$ is uniquely determined by

$$
\mathfrak{S}:=\left[\begin{array}{c}
\mathfrak{X}_{1} \\
\mathfrak{X}_{1} \mathfrak{T}_{1}
\end{array}\right]^{-1}\left[\begin{array}{c}
\mathfrak{X}_{2} \\
\mathfrak{X}_{2} \mathfrak{T}_{2}
\end{array}\right]
$$

Rewriting (2.4) as

$$
\left[\begin{array}{cc}
C & M \\
M & 0
\end{array}\right]\left[\begin{array}{c}
\mathfrak{X} \\
\mathfrak{X T}
\end{array}\right] \mathfrak{T}=\left[\begin{array}{cc}
-K & 0 \\
0 & M
\end{array}\right]\left[\begin{array}{c}
\mathfrak{X} \\
\mathfrak{X T}
\end{array}\right]
$$

we see that

$$
\mathcal{L}(\lambda) U=\left[\begin{array}{cc}
C & M \\
M & 0
\end{array}\right] U(\lambda I-\mathfrak{T})
$$


It follows that

$$
\mathcal{Q}(\lambda) \mathbf{x}=0 \Leftrightarrow(\lambda I-\mathfrak{T})\left(U^{-1}\left[\begin{array}{c}
\mathbf{x} \\
\lambda \mathbf{x}
\end{array}\right]\right)=0 .
$$

The relationship (2.9) is informative. Not only does it show that the $2 n \times 2 n$ real matrix $\mathfrak{T}$ has exactly the same spectrum as the $n \times n$ quadratic $\lambda$-matrix $\mathcal{Q}(\lambda)$, it also indicates how the associated eigenvectors of $\mathcal{Q}(\lambda)$ and $\mathfrak{T}$ can be implied from each other via the associated nonsingular matrix $U$. In this sense, we may say that the innate eigeninformation in a real standard pair $(\mathfrak{X}, \mathfrak{T})$ is equivalent to that in the original $\mathcal{Q}(\lambda)$.

We are interested in representing matrix coefficients of $\mathcal{Q}(\lambda)$ in terms of its real standard pair. Toward that end, it will be convenient to introduce the nonsingular matrix,

$$
S=S(\mathfrak{X}, \mathfrak{T}):=\left(U^{\top}\left[\begin{array}{cc}
C & M \\
M & 0
\end{array}\right] U\right)^{-1} .
$$

Note that $S$ is symmetric and is a function of the real standard pair $(\mathfrak{X}, \mathfrak{T})$. Some special choices of real standard pairs lead to special structures of $S$, which will be studied later in the paper. We now state our main result about the real-valued spectral decomposition.

Theorem 2.3. Given a real symmetric quadratic $\lambda$-matrix $\mathcal{Q}(\lambda):=M \lambda^{2}+C \lambda+K$, let $(\mathfrak{X}, \mathfrak{T})$ be a real standard pair and $S$ the corresponding nonsingular matrix defined according to (2.10). Then $\mathcal{Q}(\lambda)$ enjoys a spectral decomposition in the sense that its matrix coefficients $(M, C, K)$ can be factorized in terms of $(\mathfrak{X}, \mathfrak{T})$ as follows:

$$
\left\{\begin{aligned}
M & =\left(\mathfrak{X T} S \mathfrak{X}^{\top}\right)^{-1}, \\
C & =-M \mathfrak{X T} \mathfrak{T}^{2} S \mathfrak{X}^{\top} M, \\
K & =-M \mathfrak{X} \mathfrak{T}^{3} S \mathfrak{X}^{\top} M+C M^{-1} C .
\end{aligned}\right.
$$

Proof. By the definition of $S$, trivially we have

$$
\left[\begin{array}{c}
\mathfrak{X} \\
\mathfrak{X T}
\end{array}\right] S \mathfrak{X}^{\top} M=\left[\begin{array}{c}
0 \\
I_{n}
\end{array}\right] .
$$

The expression for $M$ immediately follows. We also have the useful fact,

$$
\mathfrak{X} S \mathfrak{X}^{\top}=0 \text {. }
$$

Post-multiplying (2.4) by $S \mathfrak{X}^{\top}$ and applying the representation of $M$ and the fact (2.13), we obtain the formula for $C$. Similarly, post-multiplying (2.4) by $\mathfrak{T} S \mathfrak{X}^{\top}$, we obtain

$$
M \mathfrak{X T}^{3} S \mathfrak{X}^{\top}+C \mathfrak{X T}^{2} S \mathfrak{X}^{\top}+K \mathfrak{X T} S \mathfrak{X}^{\top}=0 .
$$

Upon substitution by the decompositions of $M$ and $C$, the representation of $K$ follows.

We point out that our representation (2.11) is intimately related to the formula proved by Lancaster in [19, Theorem 1]. The fundamental difference is that we use only the real standard pair $(\mathfrak{X}, \mathfrak{T})$ together with the associated real matrix $S$ to represent the real symmetric matrices $(M, C, K)$, whereas Lancaster constructed the same system by employing the more general notion of Jordan triple (or Jordan pair for a self-adjoint system) that typically is complex-valued. Specifically, let $(\mathscr{X}, \mathscr{J}, \mathscr{Y})$ denote the Jordan triple referred to in [19, where $\mathscr{J}$ is a suitable Jordan matrix. Then we see that the role of matrices $\Gamma_{j}:=\mathscr{X} \mathscr{J}^{j} \mathscr{Y}, j=1,2,3$, in 
[19, Equation (6)] or [27, Theorem 7] is being replaced exactly by our $\mathfrak{X T}^{j} S \mathfrak{X}^{\top}, j=$ $1,2,3$, in (2.11). Likewise, the "self-orthogonality" of $\mathfrak{X}$ relative to $S$ as indicated in (2.13) in the above proof is analogous to the fact $\mathscr{X} \mathscr{Y}=0$ in [11, Equation $(2.12)]$ or [19, Equation (5)]. (Indeed, for a self-adjoint system, it can be made that $\mathscr{Y}=P \mathscr{X}^{*}$, where $P$ is an appropriate permutation matrix as defined in [24, Equation (18)] and is analogous to our matrix $S$.)

It is worth pointing out another useful fact. By (2.8) we see that the product,

$$
S^{-1} \mathfrak{T}=U^{\top}\left[\begin{array}{cc}
-K & 0 \\
0 & M
\end{array}\right] U
$$

is symmetric. It follows that, given a standard pair $(\mathfrak{X}, \mathfrak{T})$, the product $\mathfrak{T} S$ is symmetric. Together with the fact that $S$ is symmetric, we can prove recursively that

$$
\mathfrak{T}^{k} S=\left(\mathfrak{T}^{k} S\right)^{\top}
$$

holds for all $k=1,2, \ldots$. Actually, if we define an indefinite scalar product $[12$,

$$
[\mathbf{x}, \mathbf{y}]:=\left\langle S^{-1} \mathbf{x}, \mathbf{y}\right\rangle
$$

where $\langle\cdot, \cdot\rangle$ is the usual scalar product over $\mathbb{R}^{2 n}$, then the fact that $S^{-1} \mathfrak{T}=\mathfrak{T}^{\top} S^{-1}$ can be interpreted as $[\mathfrak{T} \mathbf{x}, \mathbf{y}]=[\mathbf{x}, \mathfrak{T} \mathbf{y}]$. In [11, Chapter S5], the transformation $\mathfrak{T}$ is said to be "symmetric" with respect to $S^{-1}$. As such, the symmetry of the powers of $\mathfrak{T}$ with respect to $S^{-1}$ is naturally expected from an operator point of view. See also [27, Section 6].

We already know that in any given real standard pair $(\mathfrak{X}, \mathfrak{T})$, the matrix $\mathfrak{T}$ alone inherits the spectrum of $\mathcal{Q}(\lambda)$. If $\mathfrak{T}$ in $(2.8)$ were a diagonal matrix, then $U$ could be thought of as the "eigenvector matrix" of the linear pencil $\mathcal{L}(\lambda)$. In view of (1.4) where the leading coefficient matrix $B$ is positive-definite, the matrix $S^{-1}$ may be considered as a "normalization factor" for the eigenvectors. In (1.4), $S^{-1}$ is taken to be the identity and, hence, the matter is simple. In our setting, it seems that the matrix $S^{-1}$ is also determined once a real standard pair is prescribed, but the following result suggests a different view.

Theorem 2.4. Let $\mathfrak{X} \in \mathbb{R}^{n \times 2 n}, \mathfrak{T} \in \mathbb{R}^{2 n \times 2 n}$ be some given matrices. If there exists a symmetric and nonsingular matrix $S \in \mathbb{R}^{n \times n}$ such that the product $\mathfrak{X} S \mathfrak{X}^{\top}$ is nonsingular and that the relationships (2.13) and (2.14) hold, then $(\mathfrak{X}, \mathfrak{T})$ is a real standard pair for the real symmetric quadratic $\lambda$-matrix $\mathcal{Q}(\lambda)$ whose matrix coefficients $M, C$ and $K$ are defined according to (2.11). Furthermore, after the construction of $(M, C, K)$, the matrix $S$ is related to $(\mathfrak{X}, \mathfrak{T})$ via (2.10).

Proof. Since $\mathfrak{X T S} \mathfrak{X}^{\top}$ is nonsingular, $M$ can be defined. By the assumption of (2.14), we see that the three matrix coefficients $M, C$ and $K$ are symmetric. By the assumption of (2.13) and the definition of $C$, we further observe that

$$
\left[\begin{array}{c}
\mathfrak{X} \\
\mathfrak{X T}
\end{array}\right]\left[\begin{array}{ll}
\mathfrak{T} S \mathfrak{X}^{T} M+S \mathfrak{X}^{\top} C & S \mathfrak{X}^{\top} M
\end{array}\right]=\left[\begin{array}{cc}
I_{n} & 0 \\
0 & I_{n}
\end{array}\right],
$$

implying that the matrix $\left[\begin{array}{c}\mathfrak{X} \\ \mathfrak{X} \mathfrak{T}\end{array}\right]$ is nonsingular. It follows that

$$
M \mathfrak{X T}^{2}\left[\begin{array}{c}
\mathfrak{X} \\
\mathfrak{X T}
\end{array}\right]^{-1}=M \mathfrak{X T}^{2}\left[\begin{array}{ll}
\mathfrak{T} S \mathfrak{X}^{T} M+S \mathfrak{X}^{\top} C & S \mathfrak{X}^{\top} M
\end{array}\right]=\left[\begin{array}{ll}
-K & -C
\end{array}\right],
$$

which is equivalent to $M \mathfrak{X T}^{2}+C \mathfrak{X T}+K \mathfrak{X}=0$. 
Theorem 2.4 can be regarded as the converse of Theorem 2.3. It is important because it does not require beforehand that the relationship (2.10) between $S$ and $(\mathfrak{X}, \mathfrak{T})$ before a real symmetric quadratic $\lambda$-matrix be defined. Rather, it qualifies whether a prescribed pair of real matrices $(\mathfrak{X}, \mathfrak{T})$ can ever serve as a standard pair at all and constructs, when feasible, the corresponding quadratic $\lambda$-matrix.

The results established is that Theorem 2.3 and Theorem 2.4 specify a necessary and sufficient condition on $(\mathfrak{X}, \mathfrak{T})$ being a real standard pair. Once a real standard pair has been identified, our theory enables us to decompose the real symmetric quadratic $\lambda$-matrix in terms of $(\mathfrak{X}, \mathfrak{T})$ and the corresponding $S$. What is needed is a real standard pair $(\mathfrak{X}, \mathfrak{T})$ other than the trivial example $\left(\mathfrak{X}_{0}, \mathfrak{T}_{0}\right)$ which, if used, gives back the quadratic $\lambda$-matrix in its own coefficients. We are particularly interested in using eigeninformation to construct a real standard pair. In the subsequent discussion, we consider some specially selected real standard pairs and study the resulting effect on the structure of the matrix $S$.

\section{Structure of $S$}

For simplicity, we shall limit our attention only to the case when $\mathfrak{T}$ is block diagonal. It seems natural to begin with this block diagonal structure because it mimics, in the real setting, the block structure of the Jordan matrix.

The following result is true in general [9, Chapter VIII, Theorem 1], regardless of whether $S$ or $\mathfrak{T}$ is derived from the context described in the preceding section or not.

Lemma 3.1. Suppose that $\mathfrak{T}=\operatorname{diag}\left\{\mathfrak{T}_{1}, \ldots, \mathfrak{T}_{k}\right\}$ where $\mathfrak{T}_{j} \in \mathbb{R}^{n_{j} \times n_{j}}$ for $j=$ $1, \ldots, k$ and that the spectra of $\mathfrak{T}_{j}$ and $\mathfrak{T}_{\ell}$ are disjoint whenever $j \neq \ell$. Then a symmetric matrix $S$ satisfies $S \mathfrak{T}^{\top}=\mathfrak{T} S$ if and only if $S=\operatorname{diag}\left\{S_{1}, \ldots, S_{k}\right\}$ where $S_{j} \in \mathbb{R}^{n_{j} \times n_{j}}$ is symmetric and $S_{j} \mathfrak{T}_{j}^{\top}=\mathfrak{T}_{j} S_{j}$ for $j=1, \ldots, k$.

Now we move into more specific details pertaining to the main thrust of this paper. We first construct a real standard pair for a real symmetric quadratic $\lambda$ matrix from its spectral data. Let the distinct eigenvalues in the spectrum of the pencil $\mathcal{Q}(\lambda)$ be denoted by

$$
\lambda_{1}, \bar{\lambda}_{1}, \lambda_{2}, \bar{\lambda}_{2}, \ldots, \lambda_{\ell}, \bar{\lambda}_{\ell}, \lambda_{\ell+1}, \ldots, \lambda_{k},
$$

where $\lambda_{1}, \ldots, \lambda_{\ell}$ are distinct complex-valued eigenvalues and $\lambda_{\ell+1}, \ldots, \lambda_{k}$ are distinct real eigenvalues, each of which has algebraic multiplicity $n_{j}$ (and, thus, $2 n_{1}+$ $\left.\cdots+2 n_{\ell}+n_{\ell+1}+\cdots+n_{k}=2 n\right)$. Associated with eigenvalue $\lambda_{j}$, let

$$
J\left(\lambda_{j}\right)=\lambda_{j} I_{n_{j}}+N_{j}
$$

denote its Jordan canonical form (which may be made of several Jordan blocks) and $X_{j}$ the $n \times n_{j}$ submatrix of corresponding generalized eigenvectors. In the above, $N_{j}$ is the $n_{j} \times n_{j}$ nilpotent matrix with at most 1's along its superdiagonal (see (3.12) for an example), depending on the geometric multiplicities of $\lambda_{j}$. It is known that the $2 n \times n_{j}$ matrix

$$
\left[\begin{array}{c}
X_{j} \\
X_{j} J\left(\lambda_{j}\right)
\end{array}\right]
$$

is of full column rank and that the equation

$$
M X_{j} J\left(\lambda_{j}\right)^{2}+C X_{j} J\left(\lambda_{j}\right)+K X_{j}=0
$$

is satisfied [11, Proposition 1.10]. 
In the event that $\lambda_{j}=\alpha_{j}+\imath \beta_{j}$ is a complex-valued eigenvalue, write

$$
X_{j}=X_{j R}+\imath X_{j I},
$$

with $X_{j R}, X_{j I} \in \mathbb{R}^{n \times n_{j}}$. We further combine the two corresponding complex-valued $n_{j} \times n_{j}$ Jordan blocks $J\left(\lambda_{j}\right)$ and $J\left(\bar{\lambda}_{j}\right)$ into one $2 n_{j} \times 2 n_{j}$ real-valued block $J_{r}\left(\lambda_{j}\right)$ defined by

$$
J_{r}\left(\lambda_{j}\right):=P_{j}^{-1}\left[\begin{array}{cc}
J\left(\alpha_{j}+\imath \beta_{j}\right) & 0 \\
0 & J\left(\alpha_{j}-\imath \beta_{j}\right)
\end{array}\right] P_{j}=\left[\begin{array}{cc}
\alpha_{j} I_{n_{j}}+N_{j} & \beta_{j} I_{n_{j}} \\
-\beta_{j} I_{n_{j}} & \alpha_{j} I_{n_{j}}+N_{j}
\end{array}\right] \text {, }
$$

where

$$
P_{j}:=\frac{1}{\sqrt{2}}\left[\begin{array}{cc}
I_{n_{j}} & -\imath I_{n_{j}} \\
I_{n_{j}} & \imath I_{n_{j}}
\end{array}\right] .
$$

Finally, we arrive at the definition,

$$
\left\{\begin{aligned}
\mathfrak{X} & :=\left[X_{1 R}, X_{1 I}, \ldots, X_{\ell R}, X_{\ell I}, X_{\ell+1}, \ldots, X_{k}\right] \\
\mathfrak{T} & :=\operatorname{diag}\left\{J_{r}\left(\lambda_{1}\right), \ldots, J_{r}\left(\lambda_{\ell}\right), J\left(\lambda_{\ell+1}\right), \ldots, J\left(\lambda_{k}\right)\right\},
\end{aligned}\right.
$$

which by construction is a real standard pair for $\mathcal{Q}(\lambda)$.

The corresponding matrix $S$ to (3.8) therefore possesses the structure as is described in Lemma 3.1. That is,

$$
S=\operatorname{diag}\left\{S_{1}, \ldots, S_{k}\right\},
$$

where all diagonal blocks $S_{j}$ are symmetric. Additionally, for $j=1, \ldots, \ell$, the matrix $S_{j} \in \mathbb{R}^{2 n_{j} \times 2 n_{j}}$ satisfies

$$
S_{j} J_{r}\left(\lambda_{j}\right)^{\top}=J_{r}\left(\lambda_{j}\right) S_{j},
$$

and for $j=\ell+1, \ldots, k$, the matrix $S_{j} \in \mathbb{R}^{n_{j} \times n_{j}}$ satisfies

$$
S_{j} J\left(\lambda_{j}\right)^{\top}=J\left(\lambda_{j}\right) S_{j} .
$$

What is interesting is that the structure of $S$ contains a far more subtle texture than at first glance, on which we elaborate in the next two subsections.

3.1. Upper Triangular Hankel Structure. Recall that an $m \times n$ matrix $H=$ $\left[h_{i j}\right]$ is said to have a Hankel structure if $h_{i j}=\eta_{i+j-1}$, where $\left\{\eta_{1}, \ldots, \eta_{m+n-1}\right\}$ are some fixed scalars. The matrix $H$ is said to be upper triangular Hankel if $\eta_{k}=0$ for all $k>\min \{m, n\}$. Note that the zero portion of an upper triangular Hankel matrix occurs at the lower right corner of the matrix.

Assume that the geometric multiplicity of $\lambda_{j}$ is $m_{j}$, that is, assume that there are $m_{j}$ Jordan blocks corresponding to the eigenvalue $\lambda_{j}$. Write

$$
N_{j}=\operatorname{diag}\left\{N_{1}^{(j)}, N_{2}^{(j)}, \ldots, N_{m_{j}}^{(j)}\right\},
$$

where $N_{i}^{(j)}$ is the nilpotent block of size $n_{i}^{(j)}$ for $i=1, \ldots, m_{j}$. A straightforward calculation shows that any symmetric solution $Z$ to the equation

$$
Z N_{j}^{\top}=N_{j} Z
$$

is necessarily of the form

$$
Z=\left[\begin{array}{cccc}
Z_{11} & Z_{12} & \ldots & Z_{1 m_{j}} \\
Z_{21} & Z_{22} & \ldots & Z_{2 m_{j}} \\
\vdots & \vdots & & \vdots \\
Z_{m_{j} 1} & Z_{m_{j} 2} & \ldots & Z_{m_{j} m_{j}}
\end{array}\right], \quad Z_{i k} \in \mathbb{R}^{n_{i}^{(j)} \times n_{k}^{(j)}},
$$


where $Z_{i k}^{\top}=Z_{k i}$ and $Z_{i k}$ is upper triangular Hankel. Equipped with this fact, we conclude from (3.11) that the matrices $S_{j}$ corresponding to the real eigenvalues $\lambda_{j}$, $j=\ell+1, \ldots, k$, are made of $m_{j} \times m_{j}$ upper triangular Hankel blocks as described in (3.14).

The very same structure persists in the following sense for the complex conjugate eigenvalues $\lambda_{j}$ when $j=1, \ldots, \ell$.

Theorem 3.2. With $J_{r}\left(\lambda_{j}\right)$ given by (3.6), a real-valued symmetric matrix $S_{j}$ satisfies (3.10) if and only if $S_{j}$ is of the form

$$
S_{j}=\left[\begin{array}{cc}
U_{j} & W_{j} \\
W_{j} & -U_{j}
\end{array}\right]
$$

where $U_{j}$ and $W_{j}$ are real-valued $n_{j} \times n_{j}$ matrices whose entries can be partitioned into upper triangular Hankel blocks of the form described in (3.14).

Proof. For simplicity, denote the blocks of $S_{j}$ in the form

$$
S_{j}=\left[\begin{array}{cc}
U & W \\
W^{\top} & V
\end{array}\right]
$$

where $U$ and $V$ are symmetric. Comparing the corresponding blocks in $S_{j} J_{r}\left(\lambda_{j}\right)^{\top}=$ $J_{r}\left(\lambda_{j}\right) S_{j}$, we obtain

$$
\begin{aligned}
N_{j} U-U N_{j}^{\top} & =\beta\left(W-W^{\top}\right), \\
N_{j} W-W N_{j}^{\top} & =-\beta(U+V), \\
N_{j} W^{\top}-W^{\top} N_{j}^{\top} & =\beta(U+V), \\
N_{j} V-V N_{j}^{\top} & =\beta\left(W-W^{\top}\right) .
\end{aligned}
$$

It follows that

$$
\begin{aligned}
N_{j}(U+V)-(U+V) N_{j}^{\top} & =2 \beta\left(W-W^{\top}\right), \\
N_{j}\left(W-W^{\top}\right)-\left(W-W^{\top}\right) N_{j}^{\top} & =-2 \beta(U+V) .
\end{aligned}
$$

Upon substituting (3.19) into (3.18), we obtain the linear equation,

$$
N_{j}^{2}\left(W-W^{\top}\right)-2 N_{j}\left(W-W^{\top}\right) N_{j}^{\top}+\left(W-W^{\top}\right) N_{j}^{2 \top}+4 \beta^{2}\left(W-W^{\top}\right)=0,
$$

which can be rewritten as

$$
\left(I_{n_{j}} \otimes N_{j}^{2}-2 N_{j} \otimes N_{j}+N_{j}^{2} \otimes I_{n_{j}}+4 \beta^{2} I_{n_{j}} \otimes I_{n_{j}}\right) \operatorname{vec}\left(W-W^{\top}\right)=0,
$$

where $\otimes$ stands for the Kronecker product and vec the column vectorization of a matrix. By the structure of $N_{j}$, we see that the coefficient matrix in (3.20) is upper triangular with constant $4 \beta^{2}$ along its diagonal. It follows that $W$ must be symmetric and, hence, $V=-U$. The equations (3.16) and (3.17) are reduced to the form (3.13). Their solutions $U$ and $W$ must be block upper triangular Hankel as is shown in (3.14). 
The above-mentioned upper triangular Hankel structure of $S$ prevails in the most general distribution of eigenvalues of $\mathcal{Q}(\lambda)$. We investigate one special case below.

3.2. Semi-simple Structure. The more generic case when all eigenvalues are semi-simple is worth studying. An eigenvalue $\lambda_{j}$ is semi-simple if its algebraic multiplicity $n_{j}$ is the same as the geometric multiplicity $m_{j}$. When all eigenvalues are semi-simple, the upper triangular Hankel structure no longer shows up. In this case, the matrix $S$ is necessarily of the form

$$
S=\operatorname{diag}\left\{\left[\begin{array}{cc}
U_{1} & W_{1} \\
W_{1} & -U_{1}
\end{array}\right], \ldots,\left[\begin{array}{cc}
U_{\ell} & W_{\ell} \\
W_{\ell} & -U_{\ell}
\end{array}\right], S_{\ell+1}, \ldots, S_{k}\right\},
$$

where $U_{j}, W_{j} \in \mathbb{R}^{n_{j} \times n_{j}}$ are symmetric for $j=1, \ldots, \ell$, and $S_{j} \in \mathbb{R}^{n_{j} \times n_{j}}$ is symmetric for $j=\ell+1, \ldots, k$. Furthermore, if all eigenvalues are simple, that is, if $n_{j}=1$ for all $j=1, \ldots, k$ in (3.1), then the matrix $S$ is of the form

$$
S=\operatorname{diag}\left\{\left[\begin{array}{cc}
s_{1} & t_{1} \\
t_{1} & -s_{1}
\end{array}\right], \ldots,\left[\begin{array}{cc}
s_{\ell} & t_{\ell} \\
t_{\ell} & -s_{\ell}
\end{array}\right], s_{\ell+1}, \ldots, s_{k}\right\},
$$

where $s_{j}, t_{j} \in \mathbb{R}$ for $j=1, \ldots, k,\left(s_{j}, t_{j}\right) \neq(0,0)$ for $j=1, \ldots, \ell$, and $s_{j} \neq 0$ for $j=\ell+1, \ldots, k$.

As a matter of fact, in the semi-simple case the structure of the matrix $S$ in (3.21) can be further simplified. It can be reduced to a structure similar to that in (3.22). We explain the procedure as follows.

Observe from (3.15) that for $j=1, \ldots, \ell$, the symmetric matrices $S_{j}$ are in fact symmetric Hamiltonian. Symmetric Hamiltonian matrices enjoy a special canonical form [1, 26]. Specifically, corresponding to each $S_{j}, j=1, \ldots, \ell$, there exists an orthogonal symplectic matrix

$$
Q_{j}=\left[\begin{array}{cc}
Q_{j 1} & Q_{j 2} \\
-Q_{j 2} & Q_{j 1}
\end{array}\right], \quad Q_{j 1}, Q_{j 2} \in \mathbb{R}^{n_{j} \times n_{j}}
$$

such that

$$
Q_{j}^{\top} S_{j} Q_{j}=\left[\begin{array}{cc}
T_{j} & 0 \\
0 & -T_{j}^{\top}
\end{array}\right]
$$

where $T_{j}$ is a diagonal matrix in $\mathbb{R}^{n_{j} \times n_{j}}$. We may assume further that

$$
T_{j}=\operatorname{diag}\left\{t_{j 1}, \ldots, t_{j n_{j}}\right\}
$$

with $t_{j \rho}<0$ for $\rho=1, \ldots, n_{j}$. With this transformation in mind, we now specify the simplest possible structure of $S$ when all eigenvalues of $\mathcal{Q}(\lambda)$ are semi-simple.

Theorem 3.3. Suppose that all eigenvalues of $\mathcal{Q}(\lambda)$ are semi-simple. Then there exists a real standard pair such that the corresponding matrix $S=\operatorname{diag}\left\{S_{1}, \ldots, S_{k}\right\}$ has the structure

$$
S_{j}= \begin{cases}\operatorname{diag}\left\{-I_{n_{j}}, I_{n_{j}}\right\}, & \text { if } \quad 1 \leq j \leq \ell \\ \operatorname{diag}\left\{\epsilon_{j 1}, \ldots, \epsilon_{j n_{j}}\right\}, & \text { if } \ell<j \leq k\end{cases}
$$

where $\epsilon_{j \rho}= \pm 1$ depending on the sign of eigenvalues of $S_{j}$.

Proof. Let $S$ initially denote the matrix defined via (2.10) in correspondence to the real standard pair $(\mathfrak{X}, \mathfrak{T})$ defined by (3.8). Our goal is to modify the real standard pair to produce the desirable structure specified in (3.26). 
For $j=1, \ldots, \ell$, let $Q_{j}$ be the Hamiltonian structure-preserving orthogonal matrix characterized in (3.24). Note that $Q_{j 1}+\imath Q_{j 2}$ is a unitary matrix in $\mathbb{C}^{n_{j} \times n_{j}}$. Columns of the complex-valued $n \times n_{j}$ matrix

$$
\widetilde{X}_{j}:=X_{j}\left(Q_{j 1}+\imath Q_{j 2}\right)\left|T_{j}\right|^{\frac{1}{2}}
$$

therefore, remain to represent eigenvectors of $\mathcal{Q}(\lambda)$ with corresponding eigenvalue $\lambda_{j}$. Based on (3.5), we can identify the real and the imaginary parts of $\widetilde{X}_{j}$ as

$$
\begin{aligned}
\tilde{X}_{j} & =\widetilde{X}_{j R}+\imath \widetilde{X}_{j I} \\
& \equiv\left[\left(X_{j R} Q_{j 1}-X_{j I} Q_{j 2}\right)\left|T_{j}\right|^{\frac{1}{2}},\left(X_{j R} Q_{j 2}+X_{j I} Q_{j 1}\right)\left|T_{j}\right|^{\frac{1}{2}}\right] \\
& =\left[X_{j R}, X_{j I}\right] Q_{j} \operatorname{diag}\left\{\left|T_{j}\right|^{\frac{1}{2}},\left|T_{j}\right|^{\frac{1}{2}}\right\} .
\end{aligned}
$$

Similarly, for $j=\ell+1, \ldots, k$, let $Q_{j}$ be the orthogonal matrix of eigenvectors of the symmetric matrix $S_{j}$ so that $Q_{j}^{\top} S_{j} Q_{j}=T_{j}:=\operatorname{diag}\left\{t_{j 1}, \ldots, t_{j n_{j}}\right\}$. Define

$$
\tilde{X}_{j}:=X_{j} Q_{j}\left|T_{j}\right|^{\frac{1}{2}},
$$

which again represents eigenvectors of $\mathcal{Q}(\lambda)$ with corresponding eigenvalue $\lambda_{j}$. It follows that $(\widetilde{\mathfrak{X}}, \mathfrak{T})$, where $\mathfrak{T}$ is the same as that given by (3.8) and

$$
\widetilde{\mathfrak{X}}:=\left[\widetilde{X}_{1 R}, \widetilde{X}_{1 I}, \ldots, \widetilde{X}_{\ell R}, \widetilde{X}_{\ell I}, \widetilde{X}_{\ell+1}, \ldots, \widetilde{X}_{k}\right],
$$

is a real standard pair. Since $N_{j}=0$ by assumption, it is easy to verify that $Q_{j}^{\top} J_{r}\left(\lambda_{j}\right) Q_{j}=J_{r}\left(\lambda_{j}\right)$ for $j=1, \ldots, \ell$ and $Q_{j}^{\top} J\left(\lambda_{j}\right) Q_{j}=J\left(\lambda_{j}\right)$ for $j=\ell+1, \ldots, k$. Through the transformation,

$$
\begin{array}{r}
Q:=\operatorname{diag}\left\{Q_{1}, \ldots, Q_{\ell}, Q_{\ell+1}, \ldots, Q_{k}\right\}, \\
D:=\operatorname{diag}\left\{T_{1}^{\frac{1}{2}}, T_{1}^{\frac{1}{2}}, \ldots, T_{\ell}^{\frac{1}{2}}, T_{\ell}^{\frac{1}{2}}, T_{\ell+1}^{\frac{1}{2}}, \ldots, T_{k}^{\frac{1}{2}}\right\},
\end{array}
$$

observe that the matrix

$$
\widetilde{S}:=D^{-1} Q^{\top} S Q D^{-1}=\left(D Q^{\top}\left[\mathfrak{X}^{\top},(\mathfrak{X T})^{\top}\right]\left[\begin{array}{cc}
C & M \\
M & 0
\end{array}\right]\left[\begin{array}{c}
\mathfrak{X} \\
\mathfrak{X T}
\end{array}\right] Q D\right)^{-1}
$$

has the structure specified in (3.26). Observe further that

$$
\begin{aligned}
\widetilde{\mathfrak{X}} & =\mathfrak{X} Q D, \\
\mathfrak{X T} Q D & =\mathfrak{X} Q \mathfrak{T} D=\mathfrak{X} Q D \mathfrak{T}=\widetilde{\mathfrak{X} T},
\end{aligned}
$$

justifying that $\widetilde{S}$ is indeed the matrix corresponding to the standard pair $(\widetilde{\mathfrak{X}}, \mathfrak{T})$.

The sign attributes characterized in the expression (3.26) are closely related to the notion of sign characteristic in the GLR theory 11, Section 10.5]. Since the idea of sign characteristic will not be used in our applications, we shall not elaborate the details in this presentation. We do make two remarks regarding the signs.

Corollary 3.4. Suppose that all eigenvalues of $\mathcal{Q}(\lambda)$ are semi-simple. Then the matrix $S$ characterized in Theorem 3.3 has trace zero.

Proof. The congruence transformation

$$
\left[\begin{array}{cc}
I_{n} & -\frac{1}{2} C M^{-1} \\
0 & I_{n}
\end{array}\right]\left[\begin{array}{cc}
C & M \\
M & 0
\end{array}\right]\left[\begin{array}{cc}
I_{n} & -\frac{1}{2} C M^{-1} \\
0 & I_{n}
\end{array}\right]^{\top}=\left[\begin{array}{cc}
0 & M \\
M & 0
\end{array}\right]
$$


asserts that the matrix $\left[\begin{array}{cc}C & M \\ M & 0\end{array}\right]$ has equal numbers of positive and negative eigenvalues. By Sylvester's law of inertia, it follows that the parameter matrix $\widetilde{S}$ defined in (3.31) has equal numbers of positive and negative 1's along its diagonal.

Finally, in contrast to (1.4) for eigenvectors of a symmetric linear pencil with positive definite leading matrix coefficient, we conclude this section with a most general orthogonality property for eigenvectors of a real symmetric quadratic $\lambda$ matrix $\mathcal{Q}(\lambda)$.

Corollary 3.5. Suppose that all eigenvalues of $\mathcal{Q}(\lambda)$ are semi-simple. Then there exists a real standard pair,

$$
\begin{aligned}
\mathfrak{X} & =\left[\mathbf{x}_{1 R}, \mathbf{x}_{1 I}, \ldots, \mathbf{x}_{\ell R}, \mathbf{x}_{\ell I}, \mathbf{x}_{2 \ell+1}, \ldots, \mathbf{x}_{2 n}\right], \\
\mathfrak{T} & =\operatorname{diag}\left\{\left[\begin{array}{cc}
\alpha_{1} & \beta_{1} \\
-\beta_{1} & \alpha_{1}
\end{array}\right], \ldots,\left[\begin{array}{cc}
\alpha_{\ell} & \beta_{\ell} \\
-\beta_{\ell} & \alpha_{\ell}
\end{array}\right], \lambda_{2 \ell+1}, \ldots, \lambda_{2 n}\right\},
\end{aligned}
$$

where $\mathbf{x}_{j R} \pm \imath \mathbf{x}_{j I}$ are complex conjugate eigenvectors associated with complex conjugate eigenvalues $\alpha_{j} \pm \imath \beta_{j}, j=1, \ldots, \ell$, and $\mathbf{x}_{j}$ is a real-valued eigenvector associated with real-valued eigenvalue $\lambda_{j}, j=2 \ell+1, \ldots, 2 n$; not all eigenvalues are necessarily distinct, such that

$$
\begin{aligned}
& {\left[\begin{array}{c}
\mathfrak{X} \\
\mathfrak{X T}
\end{array}\right]^{\top}\left[\begin{array}{cc}
C & M \\
M & 0
\end{array}\right]\left[\begin{array}{c}
\mathfrak{X} \\
\mathfrak{X T}
\end{array}\right]} \\
& \quad=\Gamma:=\operatorname{diag}\left\{\left[\begin{array}{cc}
1 & 0 \\
0 & -1
\end{array}\right], \ldots,\left[\begin{array}{cc}
1 & 0 \\
0 & -1
\end{array}\right]\right\}, \\
& {\left[\begin{array}{c}
\mathfrak{X} \\
\mathfrak{X T}
\end{array}\right]^{\top}\left[\begin{array}{cc}
-K & 0 \\
0 & M
\end{array}\right]\left[\begin{array}{c}
\mathfrak{X} \\
\mathfrak{X T}
\end{array}\right]=\Gamma \mathfrak{T} .}
\end{aligned}
$$

Proof. The expression (3.32) follows from rearranging the eigenvalues properly, if necessary, and applying Theorem 3.3 and Corollary 3.4. The expression (3.33) follows from the relationship (2.9).

The result of Corollary 3.5 can be interpreted as the simultaneous reduction of the pair of real symmetric matrices, $\left[\begin{array}{cc}C & M \\ M & 0\end{array}\right]$ and $\left[\begin{array}{cc}-K & 0 \\ 0 & M\end{array}\right]$ by real congruence. It is important to note that for any real symmetric quadratic $\lambda$-matrix $\mathcal{Q}(\lambda)$, the "canonical" matrix $\Gamma$ necessarily has equal numbers of 1 's and -1 's along its diagonal. This is in sharp contrast to (1.4) where eigenvectors are $B$-orthogonal.

\section{Applications}

Spectral decomposition of a linear transformation is so important that it has become a classic in the literature. What we have done above is to develop a theory of spectral decomposition for real symmetric quadratic $\lambda$-matrices. In particular, we realize in Theorem 2.3 that there is a matrix $S$ arising in the decomposition. The matrix $S$ plays a role of a normalization factor and, in general cases, its structure is well understood. Such knowledge can sometimes shed considerable insight into difficult problems. In this section, we describe a few applications of this theory. Some of the problems below have been discussed elsewhere in lengthy papers, but our approach significantly simplifies the arguments. 
4.1. Inverse Eigenvalue Problem. Generally speaking, an inverse eigenvalue problem is to reconstruct the coefficient matrices of a quadratic pencil from some known information of its eigenvalues and eigenvectors. Some general discussion for linear problems can be found in the book [6. The quadratic inverse eigenvalue problems are much harder. There is already a long list of studies on this subject. See, for example, 2, 16, 17, 19, 20, 21, 22, 25, 27, and the references contained therein. In this demonstration, we consider the special QIEP where the entire eigeninformation is given:

(QIEP)] Given $2 n$ eigenpairs $\left\{\left(\lambda_{j}, \mathbf{x}_{j}\right)\right\}_{j=1}^{2 n}$ with

$$
\begin{aligned}
\lambda_{2 j-1}=\bar{\lambda}_{2 j}=\alpha_{j}+\imath \beta_{j}, & \alpha_{j} \in \mathbb{R}, \beta_{j}>0, \\
\mathbf{x}_{2 j-1}=\overline{\mathbf{x}}_{2 j}=\mathbf{x}_{j R}+\imath \mathbf{x}_{j I}, \quad & \mathbf{x}_{j R}, \mathbf{x}_{j I} \in \mathbb{R}^{n}, \quad j=1,2, \ldots, \ell,
\end{aligned}
$$

and

$$
\lambda_{j} \in \mathbb{R}, \quad \mathbf{x}_{j} \in \mathbb{R}^{n}, \quad j=2 \ell+1, \ldots, 2 n,
$$

construct a real symmetric quadratic $\lambda$-matrix $\mathcal{Q}(\lambda)$ in the form of (1.1) so that the equations

$$
\lambda_{j}^{2} M \mathbf{x}_{j}+\lambda_{j} C \mathbf{x}_{j}+K \mathbf{x}_{j}=0
$$

are satisfied for all $j=1,2, \ldots, 2 n$. That is, $\mathcal{Q}(\lambda)$ has the prescribed set $\left\{\left(\lambda_{j}, \mathbf{x}_{j}\right)\right\}_{j=1}^{2 n}$ as its eigenpairs.

The system of equations in (4.1) can be written as

$$
M X \Lambda^{2}+C X \Lambda+K X=0
$$

where

$$
\begin{aligned}
X & :=\left[\mathbf{x}_{1 R}, \mathbf{x}_{1 I}, \ldots, \mathbf{x}_{\ell R}, \mathbf{x}_{\ell I}, \mathbf{x}_{2 \ell+1}, \ldots, \mathbf{x}_{2 n}\right] \\
\Lambda & :=\operatorname{diag}\left\{\left[\begin{array}{cc}
\alpha_{1} & \beta_{1} \\
-\beta_{1} & \alpha_{1}
\end{array}\right], \ldots,\left[\begin{array}{cc}
\alpha_{\ell} & \beta_{\ell} \\
-\beta_{\ell} & \alpha_{\ell}
\end{array}\right], \lambda_{2 \ell+1}, \ldots, \lambda_{2 n}\right\} .
\end{aligned}
$$

We can immediately solve this inverse problem for almost all prescribed eigeninformation by Theorems 2.3 and 2.4. Specifically, using (3.22), we have the following result.

Theorem 4.1. Assume that the matrix $\Lambda$ has only simple eigenvalues. Then the QIEP has a solution if there is a nonsingular symmetric matrix $S$ of the form

$$
S=\operatorname{diag}\left\{\left[\begin{array}{cc}
s_{1} & t_{1} \\
t_{1} & -s_{1}
\end{array}\right], \ldots,\left[\begin{array}{cc}
s_{\ell} & t_{\ell} \\
t_{\ell} & -s_{\ell}
\end{array}\right], s_{2 \ell+1}, \ldots, s_{2 n}\right\},
$$

such that $X S X^{\top}=0$ and the product $X \Lambda S X^{\top}$ is nonsingular. In this case, the matrix coefficients $M, C$ and $K$ of the solution $Q(\lambda)$ are given by

$$
M=\left(X \Lambda S X^{\top}\right)^{-1}, \quad C=-M X \Lambda^{2} S X^{\top} M, \quad K=-M X \Lambda^{3} S X^{\top} M+C M^{-1} C .
$$

Now we can be more specific by taking into account some additional physical properties. In all cases, we find it remarkable that Theorem 4.1 provides sufficient and necessary conditions for the general solution.

Example 1. For a damped vibrating system, we may assume that all the given eigenvalues in the QIEP are nonreal, that is, $\ell=n$. In this case, it might be "tempting" to try a matrix $S$ that is of simpler form, say,

$$
S=\operatorname{diag}\left\{\left[\begin{array}{cc}
1 & 0 \\
0 & -1
\end{array}\right], \ldots,\left[\begin{array}{cc}
1 & 0 \\
0 & -1
\end{array}\right]\right\}
$$


The condition that $X S X^{\top}=0$ on eigenvectors then becomes the equation

$$
X_{R} X_{R}^{\top}=X_{I} X_{I}^{\top},
$$

whereas the condition that $X \Lambda S X^{\top}$ be nonsingular becomes that the matrix

$$
\left[X_{R}, X_{I}\right]\left[\begin{array}{cc}
-\Omega & U \\
U & \Omega
\end{array}\right]\left[X_{R}, X_{I}\right]^{\top}
$$

be nonsingular with the notation

$$
\begin{aligned}
X_{R} & :=\left[\mathbf{x}_{1 R}, \ldots, \mathbf{x}_{n R}\right], \\
X_{I} & :=\left[\mathbf{x}_{1 I}, \ldots, \mathbf{x}_{n I}\right],
\end{aligned}
$$

and

$$
\begin{aligned}
\Omega & :=\operatorname{diag}\left\{\alpha_{1}, \ldots, \alpha_{n}\right\}, \\
U & :=\operatorname{diag}\left\{\beta_{1}, \ldots, \beta_{n}\right\} .
\end{aligned}
$$

We want to stress that the very brief argument above gives rise to precisely the sufficient conditions discussed in 22 for the solvability of the QIEP. Furthermore, the matrix coefficients $M, C$ and $K$ of the particular solution $\mathcal{Q}(\lambda)$ to the QIEP can be expressed as

$$
\begin{aligned}
M^{-1} & =-\left[X_{R}, X_{I}\right]\left[\begin{array}{cc}
-\Omega & U \\
U & \Omega
\end{array}\right]\left[X_{R}, X_{I}\right]^{\top}, \\
C & =M\left[X_{R}, X_{I}\right]\left[\begin{array}{cc}
U^{2}-\Omega^{2} & 2 U \Omega \\
2 U \Omega & \Omega^{2}-U^{2}
\end{array}\right]\left[X_{R}, X_{I}\right]^{\top} M, \\
K & =M\left[X_{R}, X_{I}\right]\left[\begin{array}{cc}
3 \Omega U^{2}-\Omega^{3} & 3 U \Omega^{2}-U^{3} \\
3 U \Omega^{2}-U^{3} & \Omega^{3}-3 \Omega U^{2}
\end{array}\right]\left[X_{R}, X_{I}\right]^{\top} M+C M^{-1} C .
\end{aligned}
$$

Example 2. Our quadratic $\lambda$-matrix $Q(\lambda)$ is said to be hyperbolic if $M$ is positive definite and

$$
\left(\mathbf{x}^{\top} C \mathbf{x}\right)^{2}>4\left(\mathbf{x}^{\top} M \mathbf{x}\right)\left(\mathbf{x}^{\top} K \mathbf{x}\right)
$$

for all nonzero $\mathbf{x} \in \mathbb{R}^{n}$. A hyperbolic system is said to be over-damped if, additionally, $C$ is positive definite and $K$ is positive semi-definite. For an over-damped system, it is well known that all the eigenvalues are real, nonpositive, and semisimple [11, Theorem 13.1]. This is the case that all given eigenvalues in our QIEP are real, that is, $\ell=0$. This inverse problem has been discussed in [20]. In this case, we use the matrix

$$
S=\operatorname{diag}\{I,-I\},
$$

and the condition $X S X^{\top}=0$ becomes

$$
X_{1} X_{1}^{\top}=X_{2} X_{2}^{\top},
$$

and the condition $X \Lambda S X^{\top}$ being nonsingular becomes the matrix

$$
X_{1} \Lambda_{1} X_{1}^{\top}-X_{2} \Lambda_{2} X_{2}^{\top}
$$

being nonsingular with the notation

$$
\begin{aligned}
X_{1} & :=\left[\mathbf{x}_{1}, \ldots, \mathbf{x}_{n}\right], \\
X_{2} & :=\left[\mathbf{x}_{n+1}, \ldots, \mathbf{x}_{2 n}\right],
\end{aligned}
$$

and

$$
\begin{aligned}
& \Lambda_{1}=\operatorname{diag}\left\{\lambda_{1}, \ldots, \lambda_{n}\right\}, \\
& \Lambda_{2}=\operatorname{diag}\left\{\lambda_{n+1}, \ldots, \lambda_{2 n}\right\} .
\end{aligned}
$$


Under these conditions, the corresponding matrix coefficients are then given by

$$
\begin{aligned}
M^{-1} & =X_{1} \Lambda_{1} X_{1}^{\top}-X_{2} \Lambda_{2} X_{2}^{\top}, \\
C & =M\left(X_{2} \Lambda_{2}^{2} X_{2}^{\top}-X_{1} \Lambda_{1}^{2} X_{1}^{\top}\right) M, \\
K & =M\left(X_{2} \Lambda_{2}^{3} X_{2}^{\top}-X_{1} \Lambda_{1}^{3} X_{1}^{\top}\right) M+C M^{-1} C .
\end{aligned}
$$

It has to be noted, however, that the construction thus far still cannot warrant a solution to the over-damped QIEP. We need to impose additional conditions on the prescribed spectral data to ensure that these matrix coefficients are positive definite and that the resulting $\mathcal{Q}(\lambda)$ is over-damped. One strategy exploited in 20] is based on the facts, by (4.6), that $X_{2}=X_{1} \Theta$ for some real orthogonal matrix $\Theta$ and that $\Lambda_{1}$ consists of the $n$ largest prescribed (negative) eigenvalues. A sufficient condition on $\Theta$ can then be characterized to guarantee that the reconstructed $\mathcal{Q}(\lambda)$ is over-damped [20, Corollary 6].

4.2. Total Decoupling Problem. It has been long desirable, yet with very limited success, to characterize the dynamical behavior of a complicated high-degreeof-freedom system in terms of the dynamics of some simpler low-degree-of-freedom subsystems. For linear pencils, there are usually modal coordinates under which the undamped quadratic eigenvalue problem can be represented by diagonal coefficient matrices. This amounts to the simultaneous diagonalization of two matrices by congruence or equivalence transformations [15, Section 4.5]. In other words, the undamped quadratic eigenvalue problem can be totally decoupled. Most vibrating systems, however, are quadratic and damped. It is known that three general coefficient matrices $M, C$, and $K$ cannot be diagonalized simultaneously by equivalence or congruence coordinate transformations. In the literature, engineers and practitioners have to turn to the so-called proportionally or classically damped systems for the purpose of simultaneous diagonalization.

Recently, Garvey, Friswell and Prells [7] proposed a notion that total decoupling of a system is not equivalent to simultaneous diagonalization. In particular, they argued that, under some mild assumptions, a general quadratic $\lambda$-matrix can be converted by real-valued isospectral transformations into a totally decoupled system. That is, a complicated $n$-degree-of-freedom second-order system can be reduced to $n$ totally independent single-degree-of-freedom second-order subsystems.

Given a real symmetric quadratic $\lambda$-matrix $\mathcal{Q}(\lambda)$ in the form of (1.1), the principle idea is to seek a nonsingular matrix $U \in \mathbb{R}^{2 n \times 2 n}$ such that the corresponding linearized system $\mathcal{L}(\lambda)$ defined in (2.1) is transformed into

$$
U^{\top}\left[\begin{array}{cc}
C & M \\
M & 0
\end{array}\right] U=\left[\begin{array}{cc}
C_{D} & M_{D} \\
M_{D} & 0
\end{array}\right], \quad U^{\top}\left[\begin{array}{cc}
-K & 0 \\
0 & M
\end{array}\right] U=\left[\begin{array}{cc}
-K_{D} & 0 \\
0 & M_{D}
\end{array}\right],
$$

where $M_{D}, C_{D}$ and $K_{D}$ are all diagonal matrices. Such a transformation, if it exists, is isospectral, so the original pencil (1.1) is equivalent to a totally decoupled system

$$
\mathcal{Q}_{D}(\lambda):=M_{D} \lambda^{2}+C_{D} \lambda+K_{D},
$$

in the sense that eigenvectors $\mathbf{x}$ for $\mathcal{Q}(\lambda)$ and $\mathbf{y}$ for $\mathcal{Q}_{D}(\lambda)$ are related by

$$
\left[\begin{array}{c}
\mathbf{x} \\
\lambda \mathbf{x}
\end{array}\right]=U\left[\begin{array}{c}
\mathbf{y} \\
\lambda \mathbf{y}
\end{array}\right]
$$

provided that $M_{D}$ and $M$ are nonsingular. The focus therefore is on the existence of $U$. 
The original proof of this important result by Garvey, Friswell and Prells 7 contains some ambiguities which were later clarified and simplified by Chu and del Buono in [4. A rather sophisticated algorithm was proposed in 5] for computing the transformation numerically without knowing a priori the eigeninformation. Here we offer an even simpler proof by employing the theory established in this paper.

For demonstration, assume that all the eigenvalues of $\mathcal{Q}(\lambda)$ are simple. By Corollary 3.5. we can find eigenvalues matrix $\Lambda$ and eigenvector matrix $X$ in the form of (4.3) such that

$$
\begin{aligned}
& {\left[\begin{array}{c}
X \\
X \Lambda
\end{array}\right]^{\top}\left[\begin{array}{cc}
C & M \\
M & 0
\end{array}\right]\left[\begin{array}{c}
X \\
X \Lambda
\end{array}\right]=S:=\operatorname{diag}\left\{\left[\begin{array}{cc}
1 & 0 \\
0 & -1
\end{array}\right], \ldots,\left[\begin{array}{cc}
1 & 0 \\
0 & -1
\end{array}\right]\right\}} \\
& {\left[\begin{array}{c}
X \\
X \Lambda
\end{array}\right]^{\top}\left[\begin{array}{cc}
-K & 0 \\
0 & M
\end{array}\right]\left[\begin{array}{c}
X \\
X \Lambda
\end{array}\right]=S \Lambda .}
\end{aligned}
$$

From any given

$$
M_{D}=\operatorname{diag}\left\{m_{1}, m_{2}, \ldots, m_{n}\right\}, \quad 0 \neq m_{j} \in \mathbb{R}
$$

define

$$
\begin{aligned}
C_{D} & :=\operatorname{diag}\left\{c_{1}, c_{2}, \ldots, c_{n}\right\} \\
K_{D} & :=\operatorname{diag}\left\{k_{1}, k_{2}, \ldots, k_{n}\right\}
\end{aligned}
$$

by

$$
c_{j}:= \begin{cases}-2 m_{j} \alpha_{j}, & j=1,2, \ldots, \ell \\ -m_{j}\left(\lambda_{2 j-1}+\lambda_{2 j}\right), & j=\ell+1, \ldots, n\end{cases}
$$

and

$$
k_{j}:= \begin{cases}m_{j}\left(\beta_{j}^{2}+\alpha_{j}^{2}\right), & j=1,2, \ldots, \ell \\ m_{j} \lambda_{2 j-1} \lambda_{2 j}, & j=\ell+1, \ldots, n\end{cases}
$$

Then it is readily seen that the quadratic pencil

$$
Q_{D}(\lambda):=M_{D} \lambda^{2}+C_{D} \lambda+K_{D}
$$

has the same spectrum as $Q(\lambda)$. Let $\mathbf{e}_{j}$ denote the standard $j$ th unit vector. Direct calculation shows that for $1 \leq j \leq \ell$ we have

$$
\left[\begin{array}{cc}
\mathbf{e}_{j} & 0 \\
\alpha_{j} \mathbf{e}_{j} & \beta_{j} \mathbf{e}_{j}
\end{array}\right]^{\top}\left[\begin{array}{cc}
C_{D} & M_{D} \\
M_{D} & 0
\end{array}\right]\left[\begin{array}{cc}
\mathbf{e}_{j} & 0 \\
\alpha_{j} \mathbf{e}_{j} & \beta_{j} \mathbf{e}_{j}
\end{array}\right]=\left[\begin{array}{cc}
0 & \beta_{j} m_{j} \\
\beta_{j} m_{j} & 0
\end{array}\right]
$$

and for $\ell+1 \leq j \leq n$ we have

$$
\begin{array}{r}
{\left[\begin{array}{c}
\mathbf{e}_{j} \\
\lambda_{2 j-1} \mathbf{e}_{j}
\end{array}\right]^{\top}\left[\begin{array}{cc}
C_{D} & M_{D} \\
M_{D} & 0
\end{array}\right]\left[\begin{array}{c}
\mathbf{e}_{j} \\
\lambda_{2 j-1} \mathbf{e}_{j}
\end{array}\right]} \\
{\left[\begin{array}{c}
\mathbf{e}_{j} \\
\lambda_{2 j} \mathbf{e}_{j}
\end{array}\right]^{\top}\left[\begin{array}{cc}
C_{D} & M_{D} \\
M_{D} & 0
\end{array}\right]\left[\begin{array}{c}
\mathbf{e}_{j} \\
\lambda_{2 j} \mathbf{e}_{j}
\end{array}\right]=m_{j}\left(\lambda_{2 j-1}-\lambda_{2 j}\right),}
\end{array}
$$


Obviously, we also have

$$
\frac{1}{2 m_{j} \beta_{j}}\left[\begin{array}{cc}
1 & -1 \\
1 & 1
\end{array}\right]^{\top}\left[\begin{array}{cc}
0 & \beta_{j} m_{j} \\
\beta_{j} m_{j} & 0
\end{array}\right]\left[\begin{array}{cc}
1 & -1 \\
1 & 1
\end{array}\right]=\left[\begin{array}{cc}
1 & 0 \\
0 & -1
\end{array}\right] .
$$

Now select values of $m_{j}$ such that

$$
\left\{\begin{aligned}
m_{j} \beta_{j}>0, & \text { if } 1 \leq j \leq \ell \\
m_{j}\left(\lambda_{2 j-1}-\lambda_{2 j}\right)>0, & \text { if } \ell+1 \leq j \leq n,
\end{aligned}\right.
$$

and define vectors

$$
\left\{\begin{aligned}
{\left[\mathbf{y}_{j R}, \mathbf{y}_{j I}\right]:=\frac{1}{\sqrt{2 \beta_{j} m_{j}}}\left[\mathbf{e}_{j},-\mathbf{e}_{j}\right], } & \text { if } 1 \leq j \leq \ell, \\
\mathbf{y}_{2 j-1}=\mathbf{y}_{2 j}:=\frac{1}{\sqrt{m_{j}\left(\lambda_{2 j-1}-\lambda_{2 j}\right)}} \mathbf{e}_{j}, & \text { if } \ell+1 \leq j \leq n .
\end{aligned}\right.
$$

Then it follows that

$$
\begin{gathered}
{\left[\begin{array}{c}
Y \\
Y \Lambda
\end{array}\right]^{\top}\left[\begin{array}{cc}
C_{D} & M_{D} \\
M_{D} & 0
\end{array}\right]\left[\begin{array}{c}
Y \\
Y \Lambda
\end{array}\right]=S,} \\
{\left[\begin{array}{c}
Y \\
Y \Lambda
\end{array}\right]^{\top}\left[\begin{array}{cc}
-K_{D} & 0 \\
0 & M_{D}
\end{array}\right]\left[\begin{array}{c}
Y \\
Y \Lambda
\end{array}\right]=S \Lambda,}
\end{gathered}
$$

where

$$
Y:=\left[\mathbf{y}_{1 R}, \mathbf{y}_{1 I}, \ldots, \mathbf{y}_{\ell R}, \mathbf{y}_{\ell I}, \mathbf{y}_{2 \ell+1}, \ldots, \mathbf{y}_{2 n}\right]
$$

is the eigenvector matrix of $Q_{D}(\lambda)$. Comparing (4.9) and (4.13), we conclude that the required nonsingular $2 n \times 2 n$ matrix $U$ can be taken as the matrix

$$
U=\left[\begin{array}{c}
X \\
X \Lambda
\end{array}\right]\left[\begin{array}{c}
Y \\
Y \Lambda
\end{array}\right]^{-1}
$$

It is worth noting that the technique employed above is constructive. A close examination of the procedure reveals that the assumption of simple eigenvalues is only for convenience. The approach can be extended to the "regular systems" characterized in 27, Definition 8] or high order systems. Indeed, by reordering the real eigenvalues, if necessary, we may ensure positivity of $m_{j}$ from (4.12). Consequently, the signs of $c_{j}$ and $k_{j}$ could also be determined in (4.10) and (4.11). This feature might be useful to meet the requirement of physical feasibility. Some relevant works on isospectral transformations can be found in [19, 27.

4.3. Eigenvalue Embedding Problem. Model updating concerns the modification of an existing but inaccurate model with measured data. For models characterized by a second-order dynamical system, the measured data usually involve incomplete knowledge of natural frequencies, mode shapes, or other spectral information. In conducting the updating, it is often desirable to match only the part of observed data without tampering with the other part of unmeasured or unknown eigenstructure inherent in the original model. Such an updating, if possible, is said to have no spill-over. It has been shown recently that model updating with no spillover is entirely possible for undamped quadratic systems, whereas the spill-over for damped systems generally is unpreventable [3]. Such a difficulty is sometimes compromised by considering the eigenvalue embedding problem (EEP), which we state as follows [2]: 
(EEP) Given a real symmetric quadratic $\lambda$-matrix $\mathcal{Q}_{0}(\lambda)=M_{0} \lambda^{2}$ $+C_{0} \lambda+K_{0}$ and a few of its associated eigenvalues $\left\{\lambda_{i}\right\}_{i=1}^{k}$ with $k<2 n$, assume that new eigenvalues $\left\{\mu_{i}\right\}_{i=1}^{k}$ have been measured. Update the quadratic system $\mathcal{Q}_{0}(\lambda)$ to $\mathcal{Q}(\lambda)=M \lambda^{2}+C \lambda+K$ so that only the subset $\left\{\lambda_{i}\right\}_{i=1}^{k}$ is replaced by $\left\{\mu_{i}\right\}_{i=1}^{k}$ as $k$ eigenvalues of $\mathcal{Q}(\lambda)$ while the remaining $2 n-k$ eigenpairs of $Q(\lambda)$, which usually are unknown, and all the eigenvectors are kept the same as those of the original $\mathcal{Q}_{0}(\lambda)$.

An iterative scheme that reassigns one eigenvalue at a time has been suggested in 2] as a possible numerical method for solving the EEP. That algorithm suffers from two shortcomings - that the calculation can break down prematurely, and that not all desirable eigenvalues are guaranteed to be updated. Once again, using our theory, we offer a novel approach for solving the EEP which completely circumvents all inbuilt troubles of the algorithm proposed in [2].

For demonstration, assume that all eigenvalues of $\mathcal{Q}_{0}(\lambda)$ are simple. Let the $2 n$ eigenpairs of $Q_{0}(\lambda)$ be $\left\{\left(\lambda_{i}, \mathbf{x}_{i}\right)\right\}_{i=1}^{2 n}$. Denote the real-valued representations of $\left\{\left(\lambda_{i}, \mathbf{x}_{i}\right)\right\}_{i=1}^{k}$ and $\left\{\left(\lambda_{i}, \mathbf{x}_{i}\right)\right\}_{i=k+1}^{2 n}$ as

$$
\begin{aligned}
& \Lambda_{1}:=\operatorname{diag}\left\{\left[\begin{array}{cc}
\alpha_{1} & \beta_{1} \\
-\beta_{1} & \alpha_{1}
\end{array}\right], \ldots,\left[\begin{array}{cc}
\alpha_{\ell_{1}} & \beta_{\ell_{1}} \\
-\beta_{\ell_{1}} & \alpha_{\ell_{1}}
\end{array}\right], \lambda_{2 \ell_{1}+1}, \ldots, \lambda_{k}\right\}, \\
& X_{1}:=\left[\mathbf{x}_{1 R}, \mathbf{x}_{1 I}, \ldots, \mathbf{x}_{\ell_{1} R}, \mathbf{x}_{\ell_{1} I}, \mathbf{x}_{2 \ell_{1}+1}, \ldots, \mathbf{x}_{k}\right]
\end{aligned}
$$

and

$$
\begin{aligned}
& \Lambda_{2}:=\operatorname{diag}\left\{\left[\begin{array}{cc}
\alpha_{k+1} & \beta_{k+1} \\
-\beta_{k+1} & \alpha_{k+1}
\end{array}\right], \ldots,\left[\begin{array}{cc}
\alpha_{k+\ell_{2}} & \beta_{k+\ell_{2}} \\
-\beta_{k+\ell_{2}} & \alpha_{k+\ell_{2}}
\end{array}\right], \lambda_{k+2 \ell_{2}+1}, \ldots, \lambda_{2 n}\right\}, \\
& X_{2}:=\left[\mathbf{x}_{(k+1) R}, \mathbf{x}_{(k+1) I}, \ldots, \mathbf{x}_{\left(k+\ell_{2}\right) R}, \mathbf{x}_{\left(k+\ell_{2}\right) I}, \mathbf{x}_{k+2 \ell_{2}+1}, \ldots, \mathbf{x}_{2 n}\right],
\end{aligned}
$$

respectively. Denote further the two matrices,

$$
\begin{aligned}
S_{1}^{-1}:= & {\left[\begin{array}{c}
X_{1} \\
X_{1} \Lambda_{1}
\end{array}\right]^{\top}\left[\begin{array}{cc}
C_{0} & M_{0} \\
M_{0} & 0
\end{array}\right]\left[\begin{array}{c}
X_{1} \\
X_{1} \Lambda_{1}
\end{array}\right], } \\
S_{2}^{-1}:= & {\left[\begin{array}{c}
X_{2} \\
X_{2} \Lambda_{2}
\end{array}\right]^{\top}\left[\begin{array}{cc}
C_{0} & M_{0} \\
M_{0} & 0
\end{array}\right]\left[\begin{array}{c}
X_{2} \\
X_{2} \Lambda_{2}
\end{array}\right] . }
\end{aligned}
$$

Then, by Theorem 2.3, we know that

$$
\begin{aligned}
M_{0}^{-1} & =X_{1} \Lambda_{1} S_{1} X_{1}^{\top}+X_{2} \Lambda_{2} S_{2} X_{2}^{\top}, \\
C_{0} & =-M_{0}\left(X_{1} \Lambda_{1}^{2} S_{1} X_{1}^{\top}+X_{2} \Lambda_{2}^{2} S_{2} X_{2}^{\top}\right) M_{0}, \\
K_{0} & =-M_{0}\left(X_{1} \Lambda_{1}^{3} S_{1} X_{1}^{\top}+X_{2} \Lambda_{2}^{3} S_{2} X_{2}^{\top}\right) M_{0}+C_{0} M_{0}^{-1} C_{0} .
\end{aligned}
$$

Denote the real-valued representation of $\left\{\mu_{i}\right\}_{i=1}^{k}$ by $W$. Assume that $W$ has exactly the same block diagonal structure as that of $\Lambda_{1}$, that is, assume that $W$ is of the form

$$
W=\operatorname{diag}\left\{\left[\begin{array}{rr}
\gamma_{1} & \delta_{1} \\
-\delta_{1} & \gamma_{1}
\end{array}\right], \ldots,\left[\begin{array}{rr}
\gamma_{\ell_{1}} & \delta_{\ell_{1}} \\
-\delta_{\ell_{1}} & \gamma_{\ell_{1}}
\end{array}\right], \mu_{2 \ell_{1}+1}, \ldots, \mu_{k}\right\} .
$$

Since the eigenvectors are not modified, the condition $X_{1} S_{1} X_{1}^{\top}+X_{2} S_{2} X_{2}^{\top}=0$ automatically holds. Suppose that the matrix

$$
X_{1} W S_{1} X_{1}^{\top}+X_{2} \Lambda_{2} S_{2} X_{2}^{\top}
$$


is nonsingular. Then by Theorem 2.4, we know right away that one particular solution to EEP is given by

$$
\begin{aligned}
M^{-1} & =X_{1} W S_{1} X_{1}^{\top}+X_{2} \Lambda_{2} S_{2} X_{2}^{\top} \\
C & =-M\left(X_{1} W^{2} S_{1} X_{1}^{\top}+X_{2} \Lambda_{2}^{2} S_{2} X_{2}^{\top}\right) M \\
K & =-M\left(X_{1} W^{3} S_{1} X_{1}^{\top}+X_{2} \Lambda_{2}^{3} S_{2} X_{2}^{\top}\right) M+C M^{-1} C .
\end{aligned}
$$

Combining (4.15) with (4.17), we see that the update takes place in the following way:

$$
\begin{aligned}
M^{-1} & =M_{0}^{-1}+X_{1}\left(W-\Lambda_{1}\right) S_{1} X_{1}^{\top}, \\
(4.18) C & =M\left[M_{0}^{-1} C_{0} M_{0}^{-1}-X_{1}\left(W^{2}-\Lambda_{1}^{2}\right) S_{1} X_{1}^{\top}\right] M, \\
K & =M\left[M_{0}^{-1}\left(K_{0}-C_{0} M_{0}^{-1} C_{0}\right) M_{0}^{-1}-X_{1}\left(W^{3}-\Lambda_{1}^{3}\right) S_{1} X_{1}^{\top}\right] M+C M^{-1} C,
\end{aligned}
$$

provided that $M_{0}^{-1}+X_{1}\left(W-\Lambda_{1}\right) S_{1} X_{1}^{\top}$ is nonsingular. It is critically important to note that the update formula (4.18) from $\mathcal{Q}_{0}(\lambda)$ to $\mathcal{Q}(\lambda)$ does not need the information about $\left(\Lambda_{2}, X_{2}\right)$.

We believe that this closed form solution for the EEP is of interest itself and is innovative in the literature.

\section{Conclusion}

The classical GLR theory is a powerful methodology that generalizes the notion of spectral decomposition for linear operators to matrix polynomials of arbitrary degrees. This paper modifies the GLR theory for the special application to real symmetric quadratic matrix polynomials subject to the specific restriction that all matrices in the representation be real-valued. By constructing a real standard pair in the same way described in (3.8) which includes the most general case of arbitrary algebraic or geometric multiplicities, we characterize a special type of real-valued spectral decomposition for real symmetric quadratic $\lambda$-matrices in terms of the usual eigeninformation.

In order to accommodate the requirement of a real-valued decomposition, a special matrix $S$ is introduced. Three fundamental relationships between $S \in$ $\mathbb{R}^{2 n \times 2 n}$ and $(\mathfrak{X}, \mathfrak{T}) \in \mathbb{R}^{n \times 2 n} \times \mathbb{R}^{2 n \times 2 n}$, i.e., the matrix $\mathfrak{X T} S \mathfrak{X}^{\top}$ is nonsingular, the equality $\mathfrak{X} S \mathfrak{X}^{\top}=0$ holds, and the matrix $\mathfrak{T}^{k} S$ is symmetric for all $k$, determine a necessary and sufficient condition for the spectral decomposition. The structure of $S$ is investigated under different assumptions of multiplicities. In the special case when all eigenvalues are semi-simple, $S$ can be taken to the special form

$$
S=\operatorname{diag}\left\{\left[\begin{array}{cc}
1 & 0 \\
0 & -1
\end{array}\right], \ldots,\left[\begin{array}{cc}
1 & 0 \\
0 & -1
\end{array}\right]\right\}
$$

which is the generalized notion of "orthogonality" among eigenvectors of a real symmetric quadratic matrix polynomial.

The fact that we have real-valued spectral decomposition in hand makes it convenient for application. It is demonstrated how three nontrivial inverse problems, each of which has attracted considerable research efforts in the literature, can now be easily solved by exploiting our theory. 


\section{ACKNOWLEDGMENT}

The authors are deeply indebted to Professor Peter Lancaster and two other anonymous referees for their many insightful suggestions that greatly helped us to better understand this subject.

\section{REFERENCES}

[1] A. Bunse-Gerstner, R. Byers and V. Mehrmann, A chart of numerical methods for structured eigenvalue problems, SIAM J. Matrix Anal. Appl., 13(1992), 419-453. MR1152761 (93b:65063)

[2] J. Carvalho, B. N. Datta, W. W. Lin, and C. S. Wang, Symmetric preserving eigenvalue embedding in finite element model updating of vibrating structures, J. Sound Vibration, 290(2006), 839-864. MR2196623 (2007d:74087)

[3] M. T. Chu, B. Datta, W. W. Lin and S. F. Xu, The spill-over phenomenon in quadratic model updating, AIAA J., 46(2008), 420-428.

[4] M. T. Chu and N. Del Buono, Total decoupling of a general quadratic pencil, Pt. I: Theory, J. Sound Vibration, 309(2008), 96-111.

[5] M. T. Chu and N. Del Buono, Total decoupling of a general quadratic pencil, Pt. II: Structure preserving isospectral flows, J. Sound Vibration, 309(2008), 112-128.

[6] M. T. Chu and G. H. Golub, Inverse Eigenvalue Problems; Theory, Algorithms, and Applications, Oxford University Press, Oxford, 2005. MR2263317(2007i:65001)

[7] S. D. Garvey, M. I. Friswell, and U. Prells, Coordinate transformations for general second order systems, Pt. I: General transformations, J. Sound Vibration, 258(2002), 885-909. MR1944645 (2003k:70020)

[8] S. D. Garvey, M. I. Friswell, and U. Prells, Coordinate transformations for general second order systems, Pt. II: Elementary structure preserving transformations, J. Sound Vibration, 258(2002), 911-930. MR1944646 (2003k:70021)

[9] F. R. Gantmacher, The Theory of Matrices, Vol. 1, Translated from the Russian by K. A. Hirsch, AMS Chelsea Publishing, Providence, RI, 1998. MR1657129 (99f:15001)

[10] I. Gohberg, P. Lancaster, and L. Rodman, Spectral analysis of selfadjoint matrix polynomials, Ann. of Math., 112(1980), 34-71. MR584074 (82c:15010)

[11] I. Gohberg, P. Lancaster, and L. Rodman, Matrix Polynomials, Computer Science and Applied Mathematics. Academic Press, Inc., New York, London, 1982. MR662418 (84c:15012)

[12] I. Gohberg, P. Lancaster, and L. Rodman, Matrices and Indefinite Scalar Products, Birkhaüser, Basel, 1983. MR859708 (87j:15001)

[13] P. R. Halmos, What does the spectral theorem say? Amer. Math. Monthly, 70(1963), 241-247. MR0150600 (27:595)

[14] N. J. Higham, D. S. Mackey, N. Mackey and F. Tisseur, Symmetric linearlization for matrix polynomials, SIAM J. Matrix Anal. Appl., 29(2006), 143-159. MR2288018

[15] R. A. Horn and C. R. Johnson, Matrix Analysis, Cambridge University Press, New York, 1991. MR1084815 (91i:15001)

[16] Y. C. Kuo, W. W. Lin, and S. F. Xu, Solutions of the partially described inverse quadratic eigenvalue problem, SIAM J. Matrix Anal. Appl., 29(2006), 33-53. MR2288012 (2008a:93022)

[17] P. Lancaster, Expressions for damping matrices in linear vibration problems, J. Aero-Space Sci., 28(1961), pp. 256.

[18] P. Lancaster, Lambda-matrices and Vibrating Systems, Dover Publications; Dover edition, 1966. MR0210345 (35:1238)

[19] P. Lancaster, Isospectral vibrating systems. Pt. I: The spectral method, Linear Algebra Appl., 409(2005), 51-69. MR2169546 (2006e:74043)

[20] P. Lancaster, Inverse spectral problems for semi-simple damped vibrating systems, SIAM J. Matrix Anal. Appl., 29(2007), 279-301. MR2288026

[21] P. Lancaster and J. Maroulas, Inverse problems for damped vibrating systems, J. Math. Anal. Appl., 123(1987), 238-261. MR881543 (88d:34013)

[22] P. Lancaster and U. Prells, Inverse problems for damped vibrating systems, J. Sound Vibration, 283(2005), 891-914. MR2136391 (2006a:70050)

[23] P. Lancaster and L. Rodman, Canonical forms for Hermitian matrix pairs under strict equivalence and congruence, SIAM Review, 47(2005), 407-443. MR2178635(2007i:15021) 
[24] P. Lancaster and L. Rodman, Canonical forms for symmetric/skew-symmetric real matrix pairs under strict equivalence and congruence, Linear Algebra Appl., 406(2005), 1-76. MR2156428 (2006d:15027)

[25] P. Lancaster and Q. Ye, Inverse spectral problems for linear and quadratic matrix pencils, Linear Algebra Appl., 107(1988), 293-309. MR960152 (89i:15016)

[26] C. Paige and C. Van Loan, A Schur decomposition for Hamiltonian matrices, Linear Algebra Appl., 41(1981), 11-32. MR649714 (84d:65019)

[27] U. Prells and P. Lancaster, Isospectral vibrating systems. Pt. II: Structure preserving transformations, in Operator Theory and Indefinite Inner Product Spaces, Oper. Theory Adv. Appl., 163(2006), 275-298. MR 2215867 (2006m:70043)

[28] F. Tisseur and K. Meerbergen, The quadratic eigenvalue problem, SIAM Rev., 43(2001), 253-286. MR.1861082(2002i:65042)

Department of Mathematics, North Carolina State University, Raleigh, North CarOLINA 27695-8205

E-mail address: chu@math.ncsu.edu

LMaM, School of Mathematical Sciences, Peking University, Beijing, 100871, China E-mail address: xsf@pku.edu.cn 